\title{
Non-Steroidal Anti-Inflammatory Drug (NSAID)-Induced Small Intestinal Injury
}

Akihiro Tajima*

Department of Gastroenterology, Dokkyo Medical University, Japan

\begin{abstract}
Non-steroidal anti-inflammatory drug (NSAID), including aspirin, induced small intestinal injuries are frequently seen in clinical field. Capsule endoscopy and double balloon endoscopy are major diagnostic methods. Small intestinal injury includes bleeding, erosion, and ulceration. Unfortunately, accurate mechanism(s) of NSAID-induced small intestinal injuries are remained to be determined so far. In terms of therapy, there are currently no therapies specifically designed or approved for the prevention of NSAID-induced enteropathy, although many medications are prescribed. Further clinical and basic researches are waiting.
\end{abstract}

\section{Introduction}

Nonsteroidal anti-inflammatory drugs (NSAIDs), including lowdose aspirin, are among the most widely prescribed medications, with approximately 30 million patients worldwide ingesting NSAID [1]. NSAID could cause small intestinal injury. It became clear from the studies of post-mortem samplesby Bjarnason and colleagues (1993) that NSAID use is alsoassociated with significant damage to the more distal regionsof the small intestine (i.e. distal to the ligament of Treitz) [2]. Small intestinal injury includes bleeding, erosion, and ulceration $[3,4]$. Serious complications can includemassive bleeding, perforation and strictures, sometimes leading to death [5]. PubMed with key words "NSAID and enteropathy" was used in 1994 through 2013 for selecting articles. The aim of this short review is to summarize out current understanding of NSAID induced small intestinal injury.

\section{Diagnosis of Non-Steroidal Anti-Inflammatory Drug (NSAID)-Induced Small Intestinal Injury (Table 1)}

NSAID can affect the jejunum and ileum [6]. Capsule endoscopy (CE) and double-balloon endoscopy (DBE) are diagnostic methods for visualizing small intestinal lesions [4]. In the DBE group, out of 61 patients, ulcers or erosions were observed in the ileum in six patients and in the jejunum in one patient, respectively [4]. In the cases indicated for enteroscopy, NSAIDs enteropathy occurred in half of the patients taking NSAIDs [7]. Sixteen patients used NSAID showed ulcerative lesions, and the remaining 2 patients showed diaphragm diseases. For localized lesions, 12 patients evidenced lesions in the ileum, 5 patients had lesions in the duodenum and/or jejunum, and 1 had lesions in both intestines [8]. Using CE, several studies have been reported. Post- lowdose aspirin CE detected 10 cases (50\%) with mucosal damage not apparent in baseline studies (6 cases had petechiae, 3 had erosions, and 1 had bleeding stigmata in 2 ulcers) [9]. After 2 weeks of low-dose aspirin, the percentages of subjects with small bowel pathology were $80 \%$ in the aspirin group compared with $20 \%$ in the control group $(\mathrm{p}=0.023)$ [10]. CE demonstrates evidence of macroscopic injury to the small intestine, in up to $68 \%$ of volunteers, resulting from 2 weeks ingestion of slow-release diclofenac [11]. CE found intestinal lesions in $75 \%(12 / 16)$ of patients in the chronic therapy with NSAIDs and in $11.76 \%(2 / 17)$ of controls $(\mathrm{p}<0.01)$ [6]. Small bowel injury compatible with NSAID-induced enteropathy was observed in 7/8 animals [12]. $80 \%$ patients who took aspirin or NSAID had positive results, including the presence of erosions $(n=5)$, ulceration $(n=2)$, and ulcers with early stricturing ( $\mathrm{n}=1$, diagnosed with Crohn's disease). 13.6\% took NSAIDs or aspirin, but most did not declare using these medications. Medical history would be important for the diagnosis of NSAID induced small intestinal injury [13]. Post-treatment CE identified 636 lesions in 32 of 53 subjects (60\%); including 115 denuded areas in 16 subjects, 498 erosions in 22 subjects and 23 ulcers in 8 subjects [14]. Capsule

\begin{tabular}{|c|c|c|c|c|}
\hline $\begin{array}{c}\text { Ref } \\
\text { number }\end{array}$ & $\begin{array}{l}\text { Authors } \\
\text { Year published }\end{array}$ & Methods & NSAID & Controls \\
\hline 4 & Hayashi Y (2005) & DBE & $\begin{array}{l}11.5 \% \text { (ileum/85.7\%, } \\
\text { jejunum } / 14.3 \% \text { ) }\end{array}$ & - \\
\hline 7 & Matsumoto T (2008) & DBE & $51 \%$ & $5 \%$ \\
\hline 8 & Hayashi Y ( 2009) & DBE & $\begin{array}{l}100 \% \text { (ileum } / 55.6 \% \text {, } \\
\text { duodenum and/ } \\
\text { or jejunum } / 27.8 \% \text {, } \\
\text { both } / 5.6 \% \text { ) }\end{array}$ & - \\
\hline 9 & Smecuol E (2009) & CE & $50 \%$ & - \\
\hline 10 & Endo H (2009) & CE & $80 \%$ & $20 \%$ \\
\hline 11 & Maiden L ( 2009) & CE & $68 \%$ & - \\
\hline 6 & $\begin{array}{l}\text { Caunedo-Alvarez A } \\
(2010)\end{array}$ & CE & $75 \%$ & $11.76 \%$ \\
\hline 12 & Tachecí I ( 2010) & CE & $87.50 \%$ & - \\
\hline 13 & Sidhu R (2010) & CE & $80 \%$ & - \\
\hline 14 & Fujimori S, 2010 & CE & $\begin{array}{l}60 \% \\
\text { denuded areas were } \\
\text { predominantly located in } \\
\text { the proximal part } \\
\text { erosions throughout the } \\
\text { small intestine and all } \\
\text { ulcers in the distal part }\end{array}$ & - \\
\hline 3 & Lim YJ, 2012 & CE & $55-70 \%$ & - \\
\hline
\end{tabular}

Abbreviations

CE: capsule endoscopy

DBE: double-balloon endoscopy

Table 1: Diagnosis of Non-steroidal anti-inflammatory drug (NSAID)-induced small intestinal injury.

*Corresponding author: Akihiro Tajima, Department of Gastroenterology, Dokkyo Medical University, 880 Kitakobayashi, Mibu, Shimotsuga Tochigi, 321-0293, Japan, Tel: 81-282-87-2147; Fax: 81-282-86-7761; E-mail: atajima@dokkyomed.ac.jp

Received December 09, 2013; Accepted January 13, 2014; Published January 15,2014

Citation:Tajima A (2014) Non-Steroidal Anti-Inflammatory Drug (NSAID)Induced Small Intestinal Injury. Pharm Anal Acta 5: 282. doi: 10.4172/21532435.1000282

Copyright: (C) 2014 Tajima A. This is an open-access article distributed under the terms of the Creative Commons Attribution License, which permits unrestricted use, distribution, and reproduction in any medium, provided the original author and source are credited. 
endoscopy studies have demonstrated that NSAIDs use in healthy volunteers raised the incidence (55\% to $75 \%$ ) of intestinal damage [3]. When we prescribe NSAID, we have to speculate the small intestinal lesions. Because the incidence ratio of the lesions are high (11.5-87.5\%).

\section{Probable Mechanisms of Non-Steroidal Anti- Inflammatory Drug (NSAID)-Induced Small Intestinal Injury (Table 2)}

In the stomach, inhibition of COX-1 activity leads to a rapid, compensatory increase in expression of COX-2, and suppression of both enzymes leads to exacerbation of tissue injury [2]. However, the mechanisms of NSAID-induced injury in small intestine remains to be determined so far. Many possible mechanisms have been reported. Enterobacterial translocation in the mucosa is the first step required for activation of various factors such as iNOS/NO and neutrophils, all involved in the pathogenesis of indomethacin-induced intestinal lesions

\begin{tabular}{|c|c|c|c|}
\hline $\begin{array}{l}\text { Ref } \\
\text { number }\end{array}$ & $\begin{array}{l}\text { Authors } \\
\text { Year published }\end{array}$ & Responsible candidates & $\begin{array}{l}\text { Study } \\
\text { style }\end{array}$ \\
\hline 15 & Konaka A (1999) & Enterobacterial translocation & A \\
\hline 2 & Wallace JL ( 2012) & enteropathy of enteric bacteria, and bile & $A$ \\
\hline 16 & Wallace JL ( 2011) & $\begin{array}{l}\text { PPIs exacerbate NSAID-induced } \\
\text { intestinal damage }\end{array}$ & A \\
\hline 5 & Wallace JL ( 2013) & $\begin{array}{l}\text { PPI can significantly worsen NSAID- } \\
\text { induced damage in the small intestine }\end{array}$ & A \\
\hline 17 & Takeuchi K (2010) & COX-1 and CO & A \\
\hline 18 & $\begin{array}{l}\text { Hotz-Behofsits C } \\
(2010)\end{array}$ & cox-2 & A \\
\hline 19 & Tanaka A (1999) & Nitric oxide derived by iNOS & A \\
\hline 20 & Parasher G (2001) & Nitric oxide & A \\
\hline 21 & Xue B (2009) & iNOS-dependent nitric oxide & A \\
\hline 22 & Boelsterli UA (2013) & Oxidative metabolites & A \\
\hline 23 & Omatsu T (2009) & Reactive oxygen species & $\mathrm{CL}$ \\
\hline 24 & Matsui H (2011) & mitochondrial oxidative phosphorylation & A \\
\hline 25 & Satoh H (2009) & $\begin{array}{l}\text { Insoluble dietary fibre, intestinal } \\
\text { hypermotility, leukotrienes and } \\
\text { cholinergic pathways }\end{array}$ & A \\
\hline 26 & Nandi J (2010) & TNF-alpha & A \\
\hline 27 & Shiotani A (2011) & $\begin{array}{l}\text { Combination of low-dose aspirin } \\
\text { therapy and thienopyridine }\end{array}$ & C \\
\hline 28 & $\begin{array}{l}\text { Ramirez-Alcantara V } \\
(2009)\end{array}$ & JNK & A \\
\hline 29 & Imaoka H (2010) & Reg I & A \\
\hline 30 & Kakimoto K (2010) & MMP-9 & A \\
\hline 31 & Kubo Y (2010) & $\begin{array}{l}\text { Urocortin } 1 \text { (a nonselective CRF } \\
\text { receptor agonist) }\end{array}$ & A \\
\hline 32 & Yamada S (2011) & IL-17A & A \\
\hline 33 & $\begin{array}{l}\text { Higashiyama M } \\
(2012)\end{array}$ & $\begin{array}{l}\text { Enhanced platelets-bearing neutrophil } \\
\text { migration }\end{array}$ & A \\
\hline 34 & Nadatani Y (2012) & HMGB1 & A \\
\hline 35 & Kato S (2012) & serotonin $(5-\mathrm{HT}) 3$ receptors & A \\
\hline 36 & Takagi T (2012) & Hemopexin & A \\
\hline
\end{tabular}

Abbreviations

COX-1: Cyclooxygenase

COX-2: Cyclooxygenase 2

iNOS: Inducible nitric oxide synthase

JNK: c-Jun-N-terminal kinase

Reg I: Regenerating gene

MMP-9: Matrix metalloproteinase-9

HMGB1: High mobility group box 1

A: Animal study

CL: Cell line

C: Clinical study

Table 2: Probable mechanisms of Non-steroidal anti-inflammatory drug (NSAID)induced small intestinal injury.
$[2,15]$. PPI can significantly worsen NSAID-induced damage in the small intestine $[5,16]$. Intestinal damage occurs when both COX-1 and COX-2 are inhibited, especially COX-2 $[17,18]$. Nitric oxide derived by iNOS plays a key pathogenic role in the ulcerogenic process [1921]. Oxidative metabolites that induce severe endoplasmic reticulum stress or mitochondrial stress and lead to cell death [22,23]. NSAIDs that were absorbed into the enterocytes inhibit the mitochondrial oxidative phosphorylation [24]. Insoluble dietary fibre, intestinal hypermotility, leukotrienes and cholinergic pathways are implicated in the pathogenesis of small intestinal ulcers induced by NSAIDs [25]. TNF-alpha plays an early pro-inflammatory role in indomethacininduced jejunoileitis [26]. Combination of low-dose aspirin therapy and thienopyridine may exacerbate small bowel injury [27]. Other candidate mediators of NSAID-induced small intestinal injury are JNK pathway [28], Reg I (Regenerating gene I ) [29], MMP-9 (matrix metalloproteinase-9) [30], urocortin 1(a nonselective CRF receptor agonist)[31], IL-17A [32], enhanced platelets-bearing neutrophil migration [33], HMGB1(High mobility group box 1) [34], serotonin (5-HT) 3 receptors [35], and hemopexin [36]. If the contribution of any of these candidates to NSAID-induced injury of are enough high, they would be also candidates for treatment.

\section{Prevention and Therapy of Non-Steroidal Anti- Inflammatory Drug (NSAID)-Induced Small Intestinal Injury (Table 3)}

There are currently no therapies specifically designed or approved for the prevention of NSAID-induced enteropathy [2]. Many candidates medications are summarized in Table 3. Rebamipide has not only the healing effect for NSAIDs-induced enteropathy compared with placebo [37-40]. Misoprostol have a preventive effect forNSAIDinduced small intestinal mucosal injuries [40,41]. Endogenous PGE (2) promotes the healing of small intestinal lesions $[42,43]$. Lansoprazole prevents indomethacin-induced small intestinal ulceration $[44,45]$. However opposite results have been reported later on. Omeprazole and pantoprazole cannot protect the small intestine from the damage induced by diclofenac [46,47]. Lafutidine (histamine $\mathrm{H}(2)$ receptor antagonist with a mucosal protective action) protects the small intestine against loxoprofen-induced lesions [48,49]. Satoh et al. [50] reported inhibition of acid secretion by antisecretory drugs may exacerbate NSAID-induced intestinal lesions. In terms of PPI and $\mathrm{H} 2$ receptor antagonist, the efficacy might be still controversial. Agents such as probiotics, able to modify the gut ecology, might theoretically be useful in preventing small intestinal damage induced by NSAIDs [51]. Soluble dietary fibers protect the small intestine against NSAID-induced damage $[52,53]$. Zinc was effective in protecting against indomethacininduced small intestinal damage $[54,55]$. Since endogenous NO plays a role in healing of intestinal lesions [56], NO-releasing NSAID may represent a novel class of drugs with markedly reduced intestinal toxicity [57]. Selective COX-2 inhibitors showed controversial results for small intestinal injury $[3,58]$. TNF- $\alpha$ could become a new therapeutic target for NSAID-induced small intestinal damage [59]. Highly selective pharmacologic targeting of luminal bacterial $\beta$-D-glucuronidase by a novel class of small-molecule inhibitors protects against diclofenacinduced enteropathy [60]. Mitochondrial cyclophilin D plays a key role in NSAID-induced enteropathy, lending itself as a potentially new therapeutic target for cytoprotective intervention [61]. Activation of a7 nicotinic acetylcholine receptors ameliorates indomethacininduced small intestinal ulceration [62]. Other possible medications are geranylgeranylacetone $[63,64]$, cilostazol (specific phosphodiesterase (PDE)-3 inhibitor) [33], sildenafil (inhibitor of phosphodiesterase 


\begin{tabular}{|c|c|c|c|}
\hline $\begin{array}{l}\text { Ref } \\
\text { number }\end{array}$ & $\begin{array}{l}\text { Authors } \\
\text { Year published }\end{array}$ & Candidates medications & $\begin{array}{l}\text { Report } \\
\text { style }\end{array}$ \\
\hline 38 & Fujimori S (2011) & Rebamipide & CS \\
\hline 39 & Mizukami K (2011) & Rebamipide & CS \\
\hline 37 & Kurokawa S (2013) & Rebamipide & CS \\
\hline 40 & Fujimori S (2010) & Rebamipide and misoprostol & CS \\
\hline 41 & Watanabe T (2008) & misoprostol & CS \\
\hline 43 & Arakawa T (2012) & $\begin{array}{l}\text { prostaglandin derivatives, } \\
\text { mucoprotective drugs, probiotics, and } \\
\text { mitochondrial protective drugs }\end{array}$ & A \\
\hline 42 & Takeuchi K (2010) & Endogenous PGE(2) & A \\
\hline 45 & Higuchi K (2009) & Lansoprazole & A \\
\hline 44 & Yoda Y (2010) & Lansoprazole & A \\
\hline 47 & Lim YJ (2012) & PPIs: no small bowel protective effect & A \\
\hline 46 & Zhang S (2013) & $\begin{array}{l}\text { Omeprazole and pantoprazole cannot } \\
\text { protect the small intestine from the } \\
\text { damage induced by diclofenac }\end{array}$ & A \\
\hline 48 & Amagase K (2010) & $\begin{array}{l}\text { Lafutidine (histamine } \mathrm{H}(2) \text { receptor } \\
\text { antagonist with a mucosal protective } \\
\text { action) }\end{array}$ & A \\
\hline 49 & Umegaki E (2010) & Roxatidine $(\mathrm{H}(2)$ receptor antagonist $)$ & A \\
\hline 50 & Satoh H (2012) & $\begin{array}{l}\text { Inhibition of acid secretion by } \\
\text { antisecretory drugs may exacerbate } \\
\text { NSAID-induced intestinal lesions }\end{array}$ & A \\
\hline 51 & Guslandi M (2012) & probiotics & CS \\
\hline 52 & Satoh H (2010) & Soluble dietary fibers & A \\
\hline 53 & Satoh H (2010) & $\begin{array}{l}\text { Insoluble dietary fiber and soluble } \\
\text { dietary fiber }\end{array}$ & A \\
\hline 54 & Sivalingam N (2011) & Zinc & A \\
\hline 55 & $\begin{array}{l}\text { Rodríguez de la } \\
\text { Serna A (1994) }\end{array}$ & Zinc acexamate & CS \\
\hline 57 & Wallace JL (1994) & NO-releasing NSAID & A \\
\hline 56 & Takeuchi K (2007) & Endogenous NO & A \\
\hline 3 & Lim YJ (2012) & Selective COX-2 inhibitors (coxibs) & A \\
\hline 58 & Maehata Y (2012) & $\begin{array}{l}\text { Selective COX-2 inhibitors are not } \\
\text { completely safe for the small bowel }\end{array}$ & CS \\
\hline 59 & Fukumoto K (2011) & TNF- $\alpha$ & A \\
\hline 60 & LoGuidice A (2012) & bacterial $\beta$-D-glucuronidase & A \\
\hline 61 & LoGuidice A (2010) & Mitochondrial cyclophilin D & A \\
\hline 62 & Kawahara R (2011) & a7 nicotinic acetylcholine receptors & A \\
\hline 63 & Shiotani A (2010) & geranylgeranylacetone & CS \\
\hline 64 & Iwai T (2011) & Geranylgeranylacetone & A \\
\hline 33 & $\begin{array}{l}\text { Higashiyama M } \\
(2012)\end{array}$ & $\begin{array}{l}\text { cilostazol (specific phosphodiesterase } \\
\text { (PDE)-3 inhibitor) }\end{array}$ & A \\
\hline 65 & Kato N (2009) & $\begin{array}{l}\text { Sildenafil (inhibitor of } \\
\text { phosphodiesterase subtype 5) }\end{array}$ & A \\
\hline 66 & Yasuda M (2011) & Dopamine D2-receptor antagonists & A \\
\hline 67 & Yanaka A (2013) & Sulforaphane & $\mathrm{CL}$ \\
\hline 68 & Menozzi A (2010) & K(ATP) channel opener diazoxide & A \\
\hline 69 & Marchbank T (2008) & $\begin{array}{l}\text { Natural bioactive products } \\
\text { (nutriceuticals), such as fish } \\
\text { hydrolysates }\end{array}$ & CS \\
\hline 70 & Amagase K (2012) & Monosodium glutamate & A \\
\hline 71 & Chao G (2012) & $\begin{array}{l}\text { Muscovite (natural clay consisting } \\
\text { of an insoluble double silicate of } \\
\text { aluminum and magnesium) diclofenac }\end{array}$ & A \\
\hline 72 & Davies NM (1997) & $\begin{array}{l}\text { tempo (nitroxide stable free radical } \\
\text { scavenger) and metronidazole }\end{array}$ & A \\
\hline
\end{tabular}

Abbreviations

NO: Nitric oxide

COX-2: Cyclooxygenase-2

CS: Clinical study

CR: Case report

A: Animal study

$\mathrm{CL}$ : Cell line

Table 3: Prevention and therapy of Non-steroidal anti-inflammatory drug (NSAID)induced small intestinal injury. subtype 5) [64], dopamine D2-receptor antagonists [65], sulforaphane [66], K(ATP) channel opener diazoxide [67], natural bioactive products (nutriceuticals), such as fish hydrolysates [68], monosodium glutamate [69], Muscovite (natural clay consisting of an insoluble double silicate of aluminum and magnesium) [70], and both tempo (nitroxide stable free radical scavenger) and metronidazole [71]. Further studies are necessary for selecting appropriate medication(s) for NSAID-induced small intestinal injury.

\section{Conclusion}

NSAID-induced small intestinal injuries are frequently seen in clinical field. CE and DBE are major diagnostic tools. Accurate mechanism(s) of NSAID-induced small intestinal injuries are remained to be determined so far. In terms of therapy, there are currently no therapies specifically designed or approved for the prevention of NSAID-induced enteropathy, although many medications are prescribed. Further clinical and basic researches are waiting.

\section{References}

1. Abimosleh SM, Tran CD, Howarth GS (2013) Emu oil reduces small intestinal inflammation in the absence of clinical improvement in a rat model of indomethacin-induced enteropathy. Evid Based Complement Alternat Med 2013: 429706

2. Wallace JL (2012) NSAID gastropathy and enteropathy: distinct pathogenesis likely necessitates distinct prevention strategies. Br J Pharmacol 165: 67-74.

3. Lim YJ, Yang CH (2012) Non-steroidal anti-inflammatory drug-induced enteropathy. Clin Endosc 45: 138-144.

4. Hayashi Y, Yamamoto H, Kita H, Sunada K, Sato H, et al. (2005) Non-steroidal anti-inflammatory drug-induced small bowel injuries identified by doubleballoon endoscopy. World J Gastroenterol 11: 4861-4864.

5. Wallace JL (2013) Mechanisms, prevention and clinical implications of nonsteroidal anti-inflammatory drug-enteropathy. World J Gastroenterol 19 : 1861-1876.

6. Caunedo-Alvarez A, Gómez-Rodríguez BJ, Romero-Vázquez J, ArgüellesArias F, Romero-Castro R, et al. (2010) Macroscopic small bowel mucosa injury caused by chronic nonsteroidal anti-inflammatory drugs (NSAID) use as assessed by capsule endoscopy. Rev Esp Enferm Dig 102: 80-85.

7. Matsumoto T, Kudo T, Esaki M, Yano T, Yamamoto H, et al. (2008) Prevalence of non-steroidal anti-inflammatory drug-induced enteropathy determined by double-balloon endoscopy: a Japanese multicenter study. Scand J Gastroenterol 43: 490-496.

8. Hayashi Y, Yamamoto H, Taguchi H, Sunada K, Miyata T, et al. (2009) Nonsteroidal anti-inflammatory drug-induced small-bowel lesions identified by double-balloon endoscopy: endoscopic features of the lesions and endoscopic treatments for diaphragm disease. J Gastroenterol 44: 57-63.

9. Smecuol E, Pinto Sanchez MI, Suarez A, Argonz JE, Sugai E, et al. (2009) Low-dose aspirin affects the small bowel mucosa: results of a pilot study with a multidimensional assessment. Clin Gastroenterol Hepatol 7: 524-529.

10. Endo H, Hosono K, Inamori M, Kato S, Nozaki Y, et al. (2009) Incidence of smal bowel injury induced by low-dose aspirin: a crossover study using capsule endoscopy in healthy volunteers. Digestion 79: 44-51.

11. Maiden $L$ (2009) Capsule endoscopic diagnosis of nonsteroidal antiinflammatory drug-induced enteropathy. J Gastroenterol 44: 64-71.

12. Tachecí I, Kvetina J, Bures J, Osterreicher J, Kunes M, et al. (2010) Wireless capsule endoscopy in enteropathy induced by nonsteroidal anti-inflammatory drugs in pigs. Dig Dis Sci 55: 2471-2477.

13. Sidhu R, Brunt LK, Morley SR, Sanders DS, McAlindon ME (2010) Undisclosed use of nonsteroidal anti-inflammatory drugs may underlie small-bowel injury observed by capsule endoscopy. Clin Gastroenterol Hepatol 8: 992-995.

14. Fujimori S, Gudis K, Takahashi Y, Seo T, Yamada Y, et al. (2010) Distribution of small intestinal mucosal injuries as a result of NSAID administration. Eur J Clin Invest 40: 504-510.

15. Konaka A, Kato S, Tanaka A, Kunikata T, Korolkiewicz R, et al. (1999) Roles 
of enterobacteria, nitric oxide and neutrophil in pathogenesis of indomethacininduced small intestinal lesions in rats. Pharmacol Res 40: 517-524

16. Wallace JL, Syer S, Denou E, de Palma G, Vong L, et al. (2011) Proton pump inhibitors exacerbate NSAID-induced small intestinal injury by inducing dysbiosis. Gastroenterology 141: 1314-1322.

17. Takeuchi K, Tanaka A, Kato S, Amagase K, Satoh H (2010) Roles of COX inhibition in pathogenesis of NSAID-induced small intestinal damage. Clin Chim Acta 411: 459-466.

18. Hotz-Behofsits C, Simpson RJ, Walley M, Bjarnason IT (2010) Role of COX 2 in nonsteroidal anti-inflammatory drug enteropathy in rodents. Scand J Gastroenterol 45: 822-827.

19. Tanaka A, Kunikata T, Mizoguchi H, Kato S, Takeuchi K (1999) Dual action of nitric oxide in pathogenesis of indomethacin-induced small intestinal ulceration in rats. J Physiol Pharmacol 50: 405-417.

20. Parasher G, Frenklakh L, Goodman, Siddiqui T, Nandi J, et al. (2001) Nitric oxide inhibitors ameliorate indomethacin-induced enteropathy in rats. Dig Dis Sci 46: 2536-2541.

21. Xue B, Hausmann M, Müller MH, Pesch T, Karpitschka M, et al. (2009) Afferent nerve sensitivity is decreased by an iNOS-dependent mechanism during indomethacin-induced inflammation in the murine jejunum in vitro. Neurogastroenterol Motil 21: 322-334.

22. Boelsterli UA, Redinbo MR, Saitta KS (2013) Multiple NSAID-induced hits injure the small intestine: underlying mechanisms and novel strategies. Toxicol Sci 131: 654-667.

23. Omatsu T, Naito Y, Handa O, Hayashi N, Mizushima K, et al. (2009) Involvement of reactive oxygen species in indomethacin-induced apoptosis of small intestinal epithelial cells. J Gastroenterol 44 Suppl 19: 30-34.

24. Matsui H, Shimokawa O, Kaneko T, Nagano Y, Rai K, et al. (2011) The pathophysiology of non-steroidal anti-inflammatory drug (NSAID)-induced mucosal injuries in stomach and small intestine. J Clin Biochem Nutr 48: 107-111.

25. Satoh H, Shiotani S, Otsuka N, Hatao K, Nishimura S (2009) Role of dietary fibres, intestinal hypermotility and leukotrienes in the pathogenesis of NSAIDinduced small intestinal ulcers in cats. Gut 58: 1590-1596.

26. Nandi J, Saud B, Zinkievich JM, Yang ZJ, Levine RA (2010) TNF-alpha modulates iNOS expression in an experimental rat model of indomethacininduced jejunoileitis. Mol Cell Biochem 336: 17-24.

27. Shiotani A, Honda K, Murao T, Ishii M, Fujita M, et al. (2011) Combination of low-dose aspirin and thienopyridine exacerbates small bowel injury. Scand $J$ Gastroenterol 46: 281-286.

28. Ramirez-Alcantara V, LoGuidice A, Boelsterli UA (2009) Protection from diclofenac-induced small intestinal injury by the JNK inhibitor SP600125 in a mouse model of NSAID-associated enteropathy. Am J Physiol Gastrointest Liver Physiol 297: G990-G998.

29. Imaoka H, Ishihara S, Kazumori H, Kadowaki Y, Aziz MM, et al. (2010) Exacerbation of indomethacin-induced small intestinal injuries in Reg I-knockout mice. Am J Physiol Gastrointest Liver Physiol 299: G311-G319.

30. Kakimoto K, Takai S, Murano M, Ishida K, Yoda Y, et al. (2010) Significance of chymase-dependent matrix metalloproteinase-9 activation on indomethacininduced small intestinal damages in rats. J Pharmacol Exp Ther 332: 684-689.

31. Kubo Y, Kumano A, Kamei K, Amagase K, Abe N, et al. (2010) Urocortin prevents indomethacin-induced small intestinal lesions in rats through activation of CRF2 receptors. Dig Dis Sci 55: 1570-1580.

32. Yamada S, Naito Y, Takagi T, Mizushima K, Hirai Y, et al. (2011) Reduced smallintestinal injury induced by indomethacin in interleukin-17A-deficient mice. J Gastroenterol Hepatol 26: 398-404.

33. Higashiyama M, Hokari R, Kurihara C, Ueda T, Watanabe C, et al. (2012) Indomethacin-induced small intestinal injury is ameliorated by cilostazol, a specific PDE-3 inhibitor. Scand J Gastroenterol 47: 993-1002.

34. Nadatani Y, Watanabe T, Tanigawa T, Machida H, Okazaki H, et al. (2012) High mobility group box 1 promotes small intestinal damage induced by nonsteroida anti-inflammatory drugs through Toll-like receptor 4. Am J Pathol 181: 98-110.

35. Kato S, Matsuda N, Matsumoto K, Wada M, Onimaru N, et al. (2012) Dual role of serotonin in the pathogenesis of indomethacin-induced small intestinal ulceration: pro-ulcerogenic action via $5-\mathrm{HT} 3$ receptors and anti-ulcerogenic action via 5-HT4 receptors. Pharmacol Res 66: 226-234.
36. Takagi T, Naito Y, Okada H, Takaoka M, Oya-Ito T, et al. (2012) Hemopexin is upregulated in rat intestinal mucosa injured by indomethacin. J Gastroentero Hepatol 27: 70-75.

37. Kurokawa S, Katsuki S, Fujita T, Saitoh Y, Ohta H, et al. (2013) A randomized, double-blinded, placebo-controlled, multicenter trial, healing effect of rebamipide in patients with low-dose aspirin and/or non-steroidal anti-inflammatory drug induced small bowel injury. J Gastroenterol.

38. Fujimori S, Takahashi Y, Gudis K, Seo T, Ehara A, et al. (2011) Rebamipide has the potential to reduce the intensity of NSAID-induced small intestinal injury: a double-blind, randomized, controlled trial evaluated by capsule endoscopy. $J$ Gastroenterol 46: 57-64

39. Mizukami K, Murakami K, Abe T, Inoue K, Uchida M, et al. (2011) Aspirininduced small bowel injuries and the preventive effect of rebamipide. World $\mathrm{J}$ Gastroenterol 17: 5117-5122.

40. Fujimori S, Takahashi Y, Seo T, Gudis K, Ehara A, et al. (2010) Prevention of traditional NSAID-induced small intestinal injury: recent preliminary studies using capsule endoscopy. Digestion 82: 167-172.

41. Watanabe T, Sugimori S, Kameda N, Machida H, Okazaki H, et al. (2008) Small bowel injury by low-dose enteric-coated aspirin and treatment with misoprostol: a pilot study. Clin Gastroenterol Hepatol 6: 1279-1282.

42. Takeuchi K, Tanigami M, Amagase K, Ochi A, Okuda S, et al. (2010) Endogenous prostaglandin E2 accelerates healing of indomethacin-induced small intestinal lesions through upregulation of vascular endothelial growth factor expression by activation of EP4 receptors.J Gastroenterol Hepatol 25: S67-S74.

43. Arakawa T, Watanabe T, Tanigawa T, Tominaga K, Otani K, et al. (2012) Smal intestinal injury caused by NSAIDs/aspirin: finding new from old. Curr Med Chem 19: 77-81.

44. Yoda Y, Amagase K, Kato S, Tokioka S, Murano M, et al. (2010) Prevention by lansoprazole, a proton pump inhibitor, of indomethacin -induced small intestinal ulceration in rats through induction of heme oxygenase-1. J Physiol Pharmacol 61: $287-294$

45. Higuchi K, Yoda Y, Amagase K, Kato S, Tokioka S, et al. (2009) Prevention of NSAID-Induced Small Intestinal Mucosal Injury: Prophylactic Potential of Lansoprazole. J Clin Biochem Nutr 45: 125-130.

46. Zhang S, Chao GQ, Lu B (2013) Proton pump inhibitors are not the key for therapying non-steroidal anti-inflammatory drugs-induced small intestinal injury. Rheumatol Int 33: 2513-2521.

47. Lim YJ, Phan TM, Dial EJ, Graham DY, Lichtenberger LM (2012) In vitro and in vivo protection against indomethacin-induced small intestinal injury by proton pump inhibitors, acid pump antagonists, or indomethacin-phosphatidylcholine. Digestion 86: 171-177.

48. Amagase K, Ochi A, Sugihara T, Kato S, Takeuchi K (2010) Protective effect of lafutidine, a histamine $\mathrm{H} 2$ receptor antagonist, against loxoprofen-induced small intestinal lesions in rats. J Gastroenterol Hepatol 25: S111-S118.

49. Umegaki E, Yoda Y, Tokioka S, Murano M, Higuchi K (2010) Protective effect of roxatidine against indomethacin-induced small intestinal mucosal injury in rats. J Gastroenterol Hepatol 25: S35-S40.

50. Satoh H, Amagase K, Takeuchi K (2012) Exacerbation of nonsteroidal antiinflammatory drug-induced small intestinal lesions by antisecretory drugs in rats: the role of intestinal motility. J Pharmacol Exp Ther 343: 270-277.

51. Guslandi M (2012) Non-steroidal anti-inflammatory drugs-induced smal intestinal injury and probiotic agents. World J Gastroenterol 18: 4241-4242.

52. Satoh H, Hara T, Murakawa D, Matsuura M, Takata K (2010) Soluble dietary fiber protects against nonsteroidal anti-inflammatory drug-induced damage to the small intestine in cats. Dig Dis Sci 55: 1264-1271.

53. Satoh H (2010) Role of dietary fiber in formation and prevention of small intestinal ulcers induced by nonsteroidal anti-inflammatory drug. Curr Pharm Des 16: 1209-1213.

54. Sivalingam N, Pichandi S, Chapla A, Dinakaran A, Jacob M (2011) Zinc protects against indomethacin-induced damage in the rat small intestine. Eur $\mathrm{J}$ Pharmacol 654: 106-116.

55. Rodríguez de la Serna A, Díaz-Rubio M (1994) Multicenter clinical trial of zinc acexamate in the prevention of nonsteroidal antiinflammatory drug induced gastroenteropathy. Spanish Study Group on NSAID Induced Gastroenteropathy Prevention. J Rheumatol 21: 927-933. 
Citation: Tajima A (2014) Non-Steroidal Anti-Inflammatory Drug (NSAID)-Induced Small Intestinal Injury. Pharm Anal Acta 5: 282. doi: 10.4172/2153-2435.1000282

Page 5 of 5

56. Takeuchi K, Hatazawa R, Tanigami M, Tanaka A, Ohno R, et al. (2007) Role of endogenous nitric oxide (NO) and NO synthases in healing of indomethacininduced intestinal ulcers in rats. Life Sci 80: 329-336.

57. Wallace JL, Reuter BK, Cirino G (1994) Nitric oxide-releasing non-steroida anti-inflammatory drugs: a novel approach for reducing gastrointestinal toxicity. J Gastroenterol Hepatol 9: S40-S44.

58. Maehata Y, Esaki M, Morishita T, Kochi S, Endo S, et al (2012) Small bowe injury induced by selective cyclooxygenase-2 inhibitors: a prospective, double-blind, randomized clinical trial comparing celecoxib and meloxicam. $J$ Gastroenterol 47: 387-393.

59. Fukumoto K, Naito Y, Takagi T, Yamada S, Horie R, et al. (2011) Role of tumor necrosis factor- $\alpha$ in the pathogenesis of indomethacin-induced small intestinal injury in mice. Int J Mol Med 27: 353-359.

60. LoGuidice A, Wallace BD, Bendel L, Redinbo MR, Boelsterli UA (2012) Pharmacologic targeting of bacterial $\beta$-glucuronidase alleviates nonsteroidal anti-inflammatory drug-induced enteropathy in mice. J Pharmacol Exp Ther 341: 447-454.

61. LoGuidice A, Ramirez-Alcantara V, Proli A, Gavillet B, Boelsterli UA (2010) Pharmacologic targeting or genetic deletion of mitochondrial cyclophilin $D$ protects from NSAID-induced small intestinal ulceration in mice. Toxicol Sci 118: 276-285.

62. Kawahara R, Yasuda M, Hashimura H, Amagase K, Kato S, et al. (2011) Activation of $\alpha 7$ nicotinic acetylcholine receptors ameliorates indomethacininduced small intestinal ulceration in mice. Eur J Pharmacol 650: 411-417.

63. Iwai T, Ichikawa T, Kida M, Goso Y, Kurihara M, et al. (2011) Protective effect of geranylgeranylacetone against loxoprofen sodium-induced small intestinal lesions in rats. Eur J Pharmacol 652: 121-125.
64. Kato N, Mashita Y, Kato S, Mitsufuji S, Yoshikawa T, et al. (2009) Sildenafil, an inhibitor of phosphodiesterase subtype 5 , prevents indomethacin-induced small-intestinal ulceration in rats via a NO/cGMP-dependent mechanism. Dig Dis Sci 54: 2346-2356

65. Yasuda M, Kawahara R, Hashimura H, Yamanaka N, limori M, et al (2011) Dopamine D2-receptor antagonists ameliorate indomethacin-induced smal intestinal ulceration in mice by activating a7 nicotinic acetylcholine receptors. J Pharmacol Sci 116: 274-282.

66. Yanaka A, Sato J, Ohmori S (2013) Sulforaphane protects small intestina mucosa from aspirin/NSAID-induced injury by enhancing host defense systems against oxidative stress and by inhibiting mucosal invasion of anaerobic enterobacteria. Curr Pharm Des 19: 157-162.

67. Menozzi A, Pozzoli C, Poli E, Passeri B, Gianelli P, et al. (2011) Diazoxide attenuates indomethacin-induced small intestinal damage in the rat. Eur $J$ Pharmacol 650: 378-383.

68. Marchbank T, Limdi JK, Mahmood A, Elia G, Playford RJ (2008) Clinical trial: protective effect of a commercial fish protein hydrolysate against indomethacin (NSAID)-induced small intestinal injury. Aliment Pharmacol Ther 28: 799-804.

69. Amagase K, Ochi A, Kojo A, Mizunoe A, Taue M, et al. (2012) New therapeutic strategy for amino acid medicine: prophylactic and healing promoting effect of monosodium glutamate against NSAID-induced enteropathy. J Pharmacol Sci 118: $131-137$.

70. Chao G, Zhang S (2012) Therapeutic effects of muscovite to non-steroidal antiinflammatory drugs-induced small intestinal disease. Int J Pharm 436: 154-160.

71. Davies NM, Jamali F (1997) Pharmacological protection of NSAID-induced intestinal permeability in the rat: effect of tempo and metronidazole as potential free radical scavengers. Hum Exp Toxicol 16: 345-349. 